\title{
Flexible bronchoscopy-guided microwave ablation in peripheral porcine lung: a new minimally-invasive ablation
}

\author{
Hai-Bin Yuan $^{1 \#}$, Xiang-Yu Wang ${ }^{2 \#}$, Jia-Yuan Sun ${ }^{3,4}$, Fang-Fang Xie ${ }^{3,4}$, Xiao-Xuan Zheng ${ }^{3,4}$, Guang-Yu Tao ${ }^{5}$, \\ Lei Pan $^{6}$, Douglas Kyle Hogarth ${ }^{7}$
}

${ }^{1}$ Department of Emergency, Shanghai Chest Hospital, Shanghai Jiao Tong University, Shanghai 200030, China; ${ }^{2}$ Department of Respiration, The First Affiliated Hospital of Henan University of Traditional Chinese Medicine, Zhengzhou 451200, China; ${ }^{3}$ Department of Respiratory Endoscopy and Pulmonary Medicine, Shanghai Chest Hospital, Shanghai Jiao Tong University, Shanghai 200030, China; ${ }^{4}$ Shanghai Engineering Research Center of Respiratory Endoscopy, Shanghai 200030, China; ${ }^{5}$ Department of Radiology, Shanghai Chest Hospital, Shanghai Jiao Tong University, Shanghai 200030, China; ${ }^{6}$ Department of Respiration, Shanghai Public Health Clinic Center, Fudan University, Shanghai 201058, China; ${ }^{7}$ Department of Medicine, University of Chicago Medicine, Chicago, IL, USA

Contributions: (I) Conception and design: JY Sun, HB Yuan, XY Wang; (II) Administrative support: JY Sun; (III) Provision of study materials or patients: JY Sun, XX Zheng, GY Tao, L Pan; (IV) Collection and assembly of data: FF Xie; (V) Data analysis and interpretation: HB Yuan, DK Hogarth; (VI) Manuscript writing: All authors; (VII) Final approval of manuscript: All authors.

"These authors contributed equally to this work as co-first authors.

Correspondence to: Jiayuan Sun, MD, PhD. Department of Endoscopy, Department of Pulmonary Medicine, Shanghai Chest Hospital, Shanghai Jiao Tong University, 241 West Huaihai Road, Shanghai 200030, China. Email: xkyyjysun@163.com.

Background: Transbronchial lung biopsy is an important approach to diagnose peripheral lung cancer, but bronchoscopy based treatment options are limited and poorly studied. A flexible bronchoscopy-guided water-cooled microwave ablation (MWA) catheter was developed to evaluate the feasibility and safety both in ex vivo and in vivo porcine models.

Methods: Using direct penetration of the catheter through the surface of ex vivo porcine lung, ablations ( $\mathrm{n}=9$ ) were performed at 70, 80, $90 \mathrm{~W}$ for 10 minutes. Temperatures of the catheter and 10, 15, $20 \mathrm{~mm}$ away from the tip were measured. Under bronchoscopy conditions in porcine lung, ablations ( $\mathrm{n}=18,6$ in ex vivo and 12 in vivo) were performed at $80 \mathrm{~W}$ for 5 minutes. Computed tomography (CT) was acquired perioperative, 24 hours, 2 weeks, and 4 weeks post ablation. Ablation zones were excised at 24 hours and 4 weeks respectively. Long-axis diameter (Dl) and short-axis diameter (Ds) were measured and tissues were sectioned for pathological examination.

Results: In-ex vivo lung, the temperature at $20 \mathrm{~mm}$ removed was over $60{ }^{\circ} \mathrm{C}$ at $80 \mathrm{~W}$ for $288 \pm 26$ seconds. The ablations under bronchoscopic conditions were successful in-ex vivo and in vivo lung. No complications occurred during the procedures. Coagulation necrosis was visible at 24 hours, and repaired fibrous tissue was seen at 4 weeks.

Conclusions: The flexible bronchoscopy-guided water-cooled MWA is feasible and safe. This early animal data holds promise of MWA becoming a potential therapeutic tool for Peripheral Lung Cancers.

Keywords: Peripheral lung; bronchoscopy; microwave ablation (MWA); feasibility; safety

Submitted Jul 11, 2019. Accepted for publication Oct 12, 2019.

doi: $10.21037 /$ tlcr.2019.10.12

View this article at: http://dx.doi.org/10.21037/tlcr.2019.10.12 


\section{Introduction}

Lung cancer is the leading cause of cancer death in the world (1). Surgical resection is the standard cure for patients with stage I or II non-small-cell lung cancer (NSCLC). However, a significant number of patients with NSCLC are not candidates for surgery due to severe medical complications, primarily associated with deteriorated lung function (2). Minimal invasive therapies including percutaneous image-guided ablation and stereotactic body radiation therapy (SBRT) have been developed to give an option for curative treatment in early stage patients (3). However, percutaneous approaches can develop difficult complications [pneumothorax (PTX), fistula] and SBRT can induce radiation pneumonitis. In this poor functioning group, complications like this affect quality of life and show modest improvement in mortality (4).

Image-guided minimal invasive ablative therapies are delivered by using needlelike applicators including hightemperature ablation (such as radiofrequency, microwave and laser), chemical ablation, cryoablation and irreversible electroporation (5-7). Radiofrequency ablation (RFA) and microwave ablation (MWA) have larger evidence and experience. MWA has several theoretical advantages over RFA in producing higher temperatures and larger volumes, less ablation time, less dependence on the electrical conductivities of tissue, and is less limited by the rising electrical impedance of tumor tissue (8-10). MWA functions by destroying tumor through the application of high temperature generated by electromagnetic energy to rapidly rotate adjacent polar water molecules within the targeted pathologic tissue, which leads to protein denaturation, cell membrane disruption, and finally coagulation necrosis with cellular death (10). These changes appear within 4-6 minutes of treatment at a temperature greater than $50{ }^{\circ} \mathrm{C}$ and occur almost immediately at $60{ }^{\circ} \mathrm{C}(8,11)$. Percutaneous use of MWA has been widely used in liver cancer therapy (12), and has become the preferred energy modality in the percutaneous ablation of air-filled pulmonary neoplasms, particularly for big lesions or those close to large vessels which may cause rapid dissipation of the applied heat via "heart sinks" $(8,13,14)$. Also internally cooled applicator had been used to cause larger coagulation necrosis and avoid unnecessary damage to the surrounding tissue (11). Lung ablation therapy is commonly performed percutaneously under image-guidance from computed tomographic (CT) or ultrasound. The incidence of PTX (occurring in 15\% of patients, $6 \%$ of which require chest tube placement) is similar to that of percutaneous lung biopsy, indicating no relation to thermal ablation itself $(15,16)$. With the development of several guided-bronchoscopy technologies, the procedural risk for PTX is lower than that under CT image-guidance (occurring in $<2 \%$ of patient, $<1 \%$ of which required chest tube placement) (17). A new approach that combines the high efficiency of MWA and a favorable safety profile of bronchoscopy is needed.

Bronchoscopy-guided, internally water-cooled MWA utilizes a new flexible microwave antenna which fits through the working channel of a therapeutic bronchoscope with enough length to reach the lung lesion invading the natural bronchial lumen. There used to be a flexible bronchoscopically-guided, gas $\left(\mathrm{CO}_{2}\right)$-cooled MWA antenna (Neuwave, Madison, WI, USA) (18). The difference was the cooling method, water-cooling was readily available and stored in the clinic while gas-cooling had a higher cooling efficiency.

In this study, we describe a new approach of lung ablation using a flexible water-cooled MWA under bronchoscopy and evaluate its feasibility and safety in both ex vivo and in vivo animal models (lung).

\section{Methods}

The study protocol was approved by the Ethics Committee at Shanghai Chest Hospital (Shanghai, China) (KS1610).

All studies were performed by using the microwave platform (KY-2000, Canyon Medical Inc., Nanjing, China). The microwave unit consists of a $2.45 \mathrm{GHz}$ generator, a flexible coaxial cable, a water-pumping machine and a flexible bronchoscopy-guided, internally water-cooled MWA antenna (Canyon Medical Inc, Patent 201620582789.0) with a diameter of $1.90 \mathrm{~mm}$ and a length of $1,150 \mathrm{~mm}$, and a $7 \mathrm{~mm}$ long stainless tip (Figure $1 A, 1)$. Inside the cooled-catheter, there are dual channels in which $4{ }^{\circ} \mathrm{C}$ physiological saline water is continuously extracted, circulated and discharged with $42 \mathrm{~mL}$ per minute flow rate. The temperature of the catheter is monitored through a thermocouple measuring the circulation water pumped-out in real time. To simulate in vivo conditions, all ex vivo tissues were perfused with $37^{\circ} \mathrm{C}$ saline water.

\section{Ablation by antenna penetrated through the surface of normal ex vivo lung}

In study 1 , five fresh ex vivo porcine lungs were collected and uninflated. The antenna was directly penetrated at least 

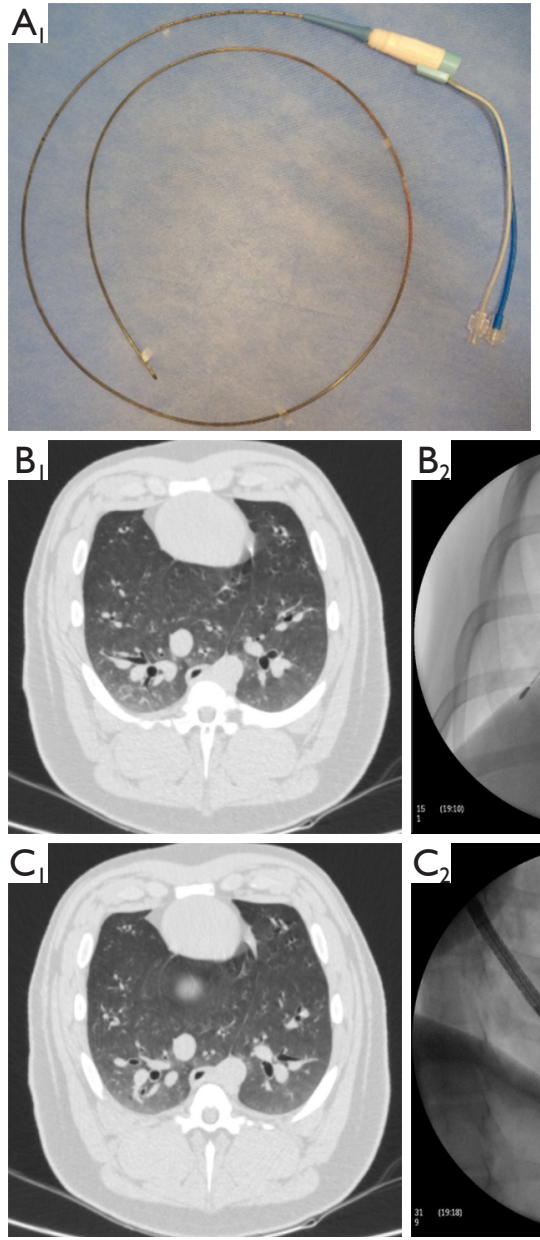
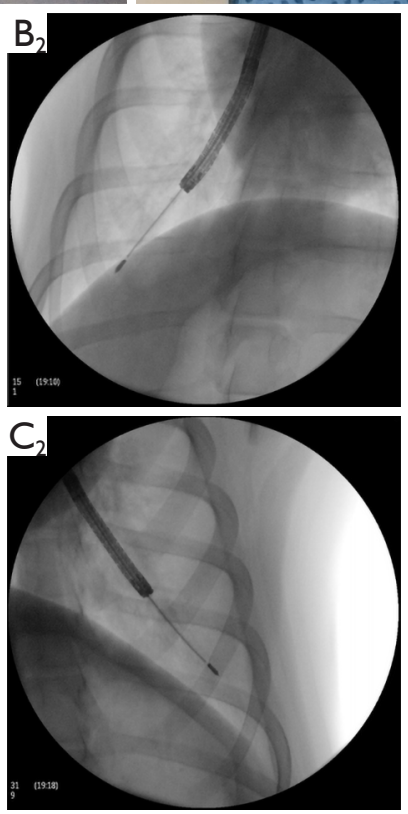
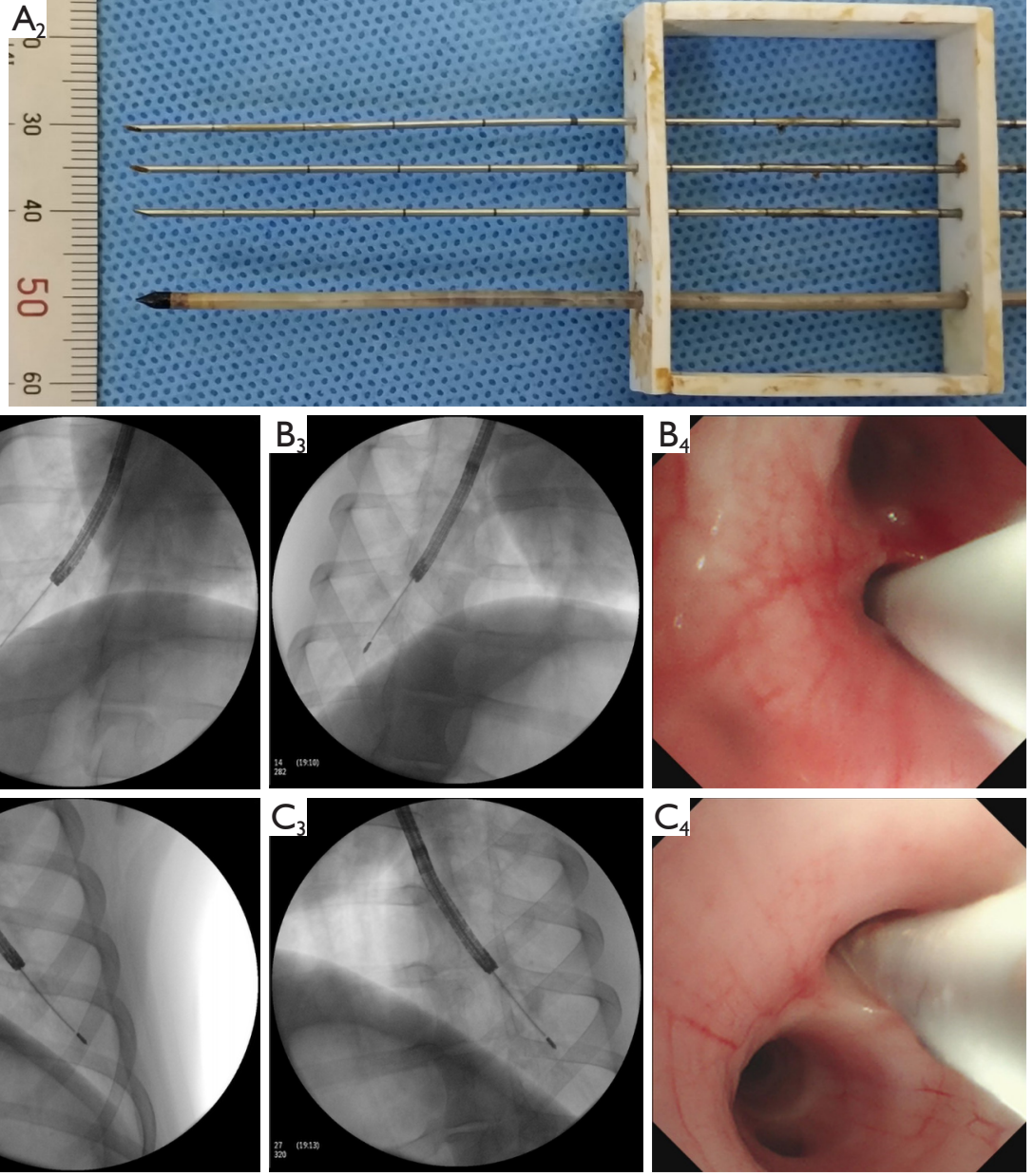

Figure 1 The model of MWA antenna, the method of measuring temperature in-ex vivo and the guiding image of operating in vivo. (A1) Prototype flexible bronchoscopy-guided, water-cooled MWA antenna. The outer-diameter of catheter: $1.90 \mathrm{~mm}$; length: 1,150 mm; the tip: 7-mm long stainless steel. (A2) The arrangement of probes for measuring temperature in ex vivo models. The antenna was stabilized with thermocouples at $5 \mathrm{~mm}$ intervals to measure the temperature ex vivo. In right (B1-4) and left (C1-4), MWA antenna was inserted in the second dorsolateral branch of the septal bronchus in vivo. The right (B1) and left (C1) target bronchus on CT in vivo. The right (B2) and left (C2) posterior-anterior position of the C-arm, illustrate the catheter and tip. The right (B3) and left (C3) oblique position of the C-arm, illustrate the catheter and tip. Under bronchoscopy guidance, the antenna was inserted into the right (B4) and left (C4) distal branch and the catheter was visible. MWA, microwave ablation.

$5 \mathrm{~cm}$ from the pleural surface. Ablations were set at 70, 80, $90 \mathrm{~W}$ for 10 minutes respectively. A total of 9 ablations were performed (three repetitions per power). Temperatures of three sites $(10,15$, and $20 \mathrm{~mm}$ peripheral to the heating zone) were measured by thermocouples parallel away from the antenna tip with $5 \mathrm{~mm}$ intervals (Figure 1A, 2). Temperature-time curves were calculated and the total time of temperature up to $60^{\circ} \mathrm{C}$ at site $20 \mathrm{~mm}$ were evaluated.

\section{Ablation by antenna through the working channel of bronchoscopy in ex vivo and in vivo lung}

In study 2, three fresh ex vivo porcine lungs were collected and inflated under the control of ventilator (SAVINA, Dräger, Germany) with a tracheal intubation. Synchronized intermittent mandatory ventilation (SIMV) mode, tidal volume (VT) $500 \mathrm{~mL}$, inspiratory time (Tinsp) 1.6 seconds (s), frequency (F) 14 breath per minute (bpm), inspiratory 
pressure (Pinsp) $14 \mathrm{mBar}$, positive end-expiratory pressure (PEEP) $4 \mathrm{mBar}$ were set. The flexible MWA antenna covered by a protection tube [extended working channel (EWC), inReach system, Covidien, America] safely pass the working channel of a flexible video-bronchoscope (model 1T-260; biopsy channel diameter $2.8 \mathrm{~mm}$; Olympus; Tokyo, Japan). This set of apparatus was accurately located to the second dorsolateral branch of the septal bronchus in bilateral lung. The optimal parameter, $80 \mathrm{~W}$ for 5 minutes was used. A total of 6 ablations were performed with three repetitions per lobe. Lesion D1, Ds were measured and Volume $\left(\mathrm{Vol}=1 / 6 \times \pi \times \mathrm{Dl} \times \mathrm{Ds}^{2}\right)$ was calculated (19).

In study 3 , six pigs weighing 60-65 kg each were sedated with $200 \mathrm{mg}$ of Xylazine Hydrochloride (Huamu Animal Medical Inc., Jilin, China) intramuscularly and anesthesia was maintained with $4-6 \mathrm{mg} / \mathrm{kg} / \mathrm{hour}$ of propofol (Hospira Inc., Lake Forest, USA) intravenously after fasting overnight. All animals were scanned by CT (Philips Brilliance 64 multi-slice spiral CT, Philips Healthcare, The Netherlands) with 0.67 -mm-thick contiguous transverse computed tomographic sections. Virtual bronchoscopic navigation (VBN; DirectPath; Olympus, Tokyo, Japan) were used for assistance in navigation. Tracheal intubation was inserted and breathing under the control of ventilator (SAVINA, Dräger). The flexible MWA antenna covered by EWC, was guided to the target bronchus through the working channel of the bronchoscope (1T-260, Olympus). The procedure was fluoroscopically-guided by the C-arm (Philips Veradius Unity, Philips Healthcare, The Netherlands). The positions of ablation were located to the second dorsolateral branch of the septal bronchus on CT and the orientation of the tip was adjusted towards the target, at least $10 \mathrm{~mm}$ away from pleura confirmed with multiple fluoroscopy projections in right (Figure $1 B$ ) and left (Figure 1C) lung, representatively shown. The ablation parameter, $80 \mathrm{~W}$ for 5 minutes, was set. Continuously electrocardiography and pulse oximetry were monitoring. Twelve total ablations were performed. After the operation, a CT scan was performed immediately. All ablations were divided into groups based on sacrifice time (group A, 24 hours, n=6; group B, 4 weeks, n=6). Additional CT scan and bronchoscopy were performed at intervals of 24 hours (12/12 ablations), 2 weeks (6/12 ablations), 4 weeks (6/12 ablations). Two maximal dimensions in both axial and coronal plane were measured for lesions of CT images by using ruler, as previously described $(19,20)$. The ablation zones dissected were along the axis of bronchus where the antenna inserted and Dl, Ds were measured. Each vivo ablated zone was then stored in $10 \%$ formalin for paraffin sectioning, hematoxylin-eosin (HE) staining, Terminal deoxynucleotidyl transferase mediated dUTP nick end labeling (TUNEL) staining assay, Masson trichrome staining for microscopic study were performed by pathologists, as previously described $(21,22)$.

\section{Statistical analysis}

Results were presented as mean \pm SD and analyzed using SPSS version 20.0 statistical software (IBM, New York, United States). Comparisons between groups were performed by $t$-test. A $\mathrm{P}$ value $<0.05$ was considered significant.

\section{Results}

In study 1 , temperature of site $10 \mathrm{~mm}, 15 \mathrm{~mm}$ at 70,80 , $90 \mathrm{~W}$ could reach the effective temperature needed for ablation. At site $20 \mathrm{~mm}$ only the power settings of 80 and $90 \mathrm{~W}$ could touch the effective temperature and the active time of $80 \mathrm{~W}$ was $288 \pm 26 \mathrm{~s}, 90 \mathrm{~W}$ was $216 \pm 39 \mathrm{~s}$ (Figure 2).

In study 2 and 3, all ablation lesions $(n=18,6$ in ex vivo and 12 in vivo) dissected from the porcine lung could be observed macroscopically. In ex vivo lung, the mean Dl was $22.7 \pm 2.3 \mathrm{~mm}$ and mean Ds was $15.0 \pm 1.5 \mathrm{~mm}$. In vivo, the mean Dl of 24 hours was $19.3 \pm 2.7 \mathrm{~mm}$ and mean Ds of 24 hours was $13.2 \pm 1.0 \mathrm{~mm}$, while the mean Dl of 4 weeks was $6.8 \pm 2.6 \mathrm{~mm}$ and mean Ds of 4 weeks was $4.7 \pm 1.5 \mathrm{~mm}$. The length of the gross observation varies in ex vivo and in vivo at different time $(\mathrm{P}<0.01)$ (Figure 3).

No major complications (e.g., PTX and pulmonary parenchymal hemorrhage) occurred during the procedure and the post-procedural period. Common CT findings after immediate ablation were areas of ground glass opacity (GGO) around the area of antenna activity. After an initial increase in 24 hours, there was a persistent reduction in diameter of the ablated areas at subsequent examinations, consistent with consolidation of the pulmonary parenchyma. Most lesions were nodular at 4 weeks on CT (Figure 4).

\section{Histopathology assessment}

\section{Twenty-four hours after ablation (group A)}

The ablation zone was centered along the bronchus at the area of antenna insertion. Under HE staining, a succession of three concentric layers with different characteristics were seen around the bronchus. The bronchial mucosa (BM) and 

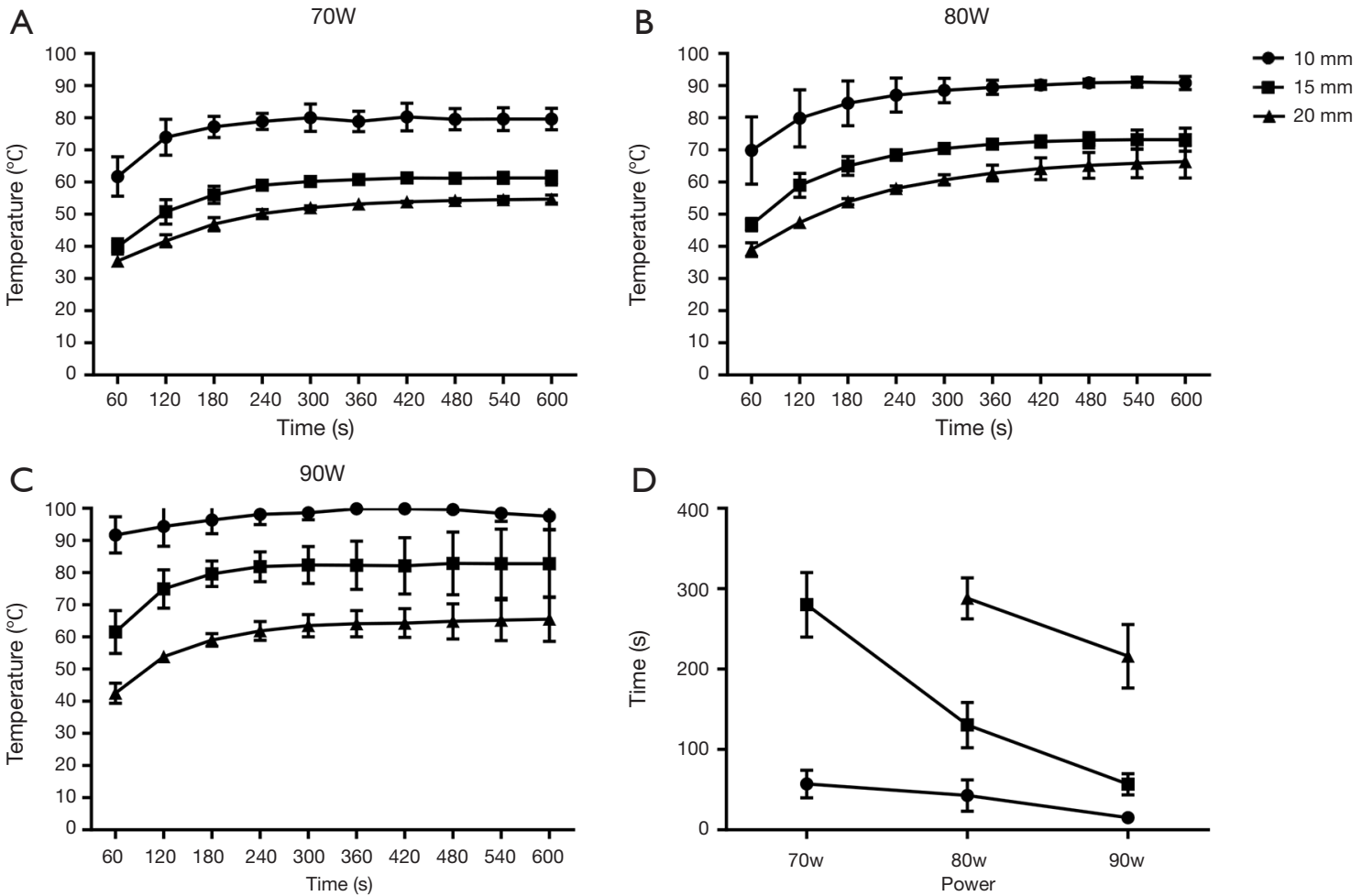

Figure 2 Temperature and time curves for ex vivo porcine lung ablation by microwave antenna penetrated through the surface. At each power setting $70 \mathrm{~W}(\mathrm{~A}), 80 \mathrm{~W}(\mathrm{~B}), 90 \mathrm{~W}(\mathrm{C})$, the curves show the temperature changes at 10, 15, and 20 mm away from the tip of antenna. (D) Time spent at the effective temperature $\left(\geq 60^{\circ} \mathrm{C}\right)$ for ablation for each site $(10,15$, and $20 \mathrm{~mm}$ from target) and power setting $(70,80$, and $90 \mathrm{~W})$.
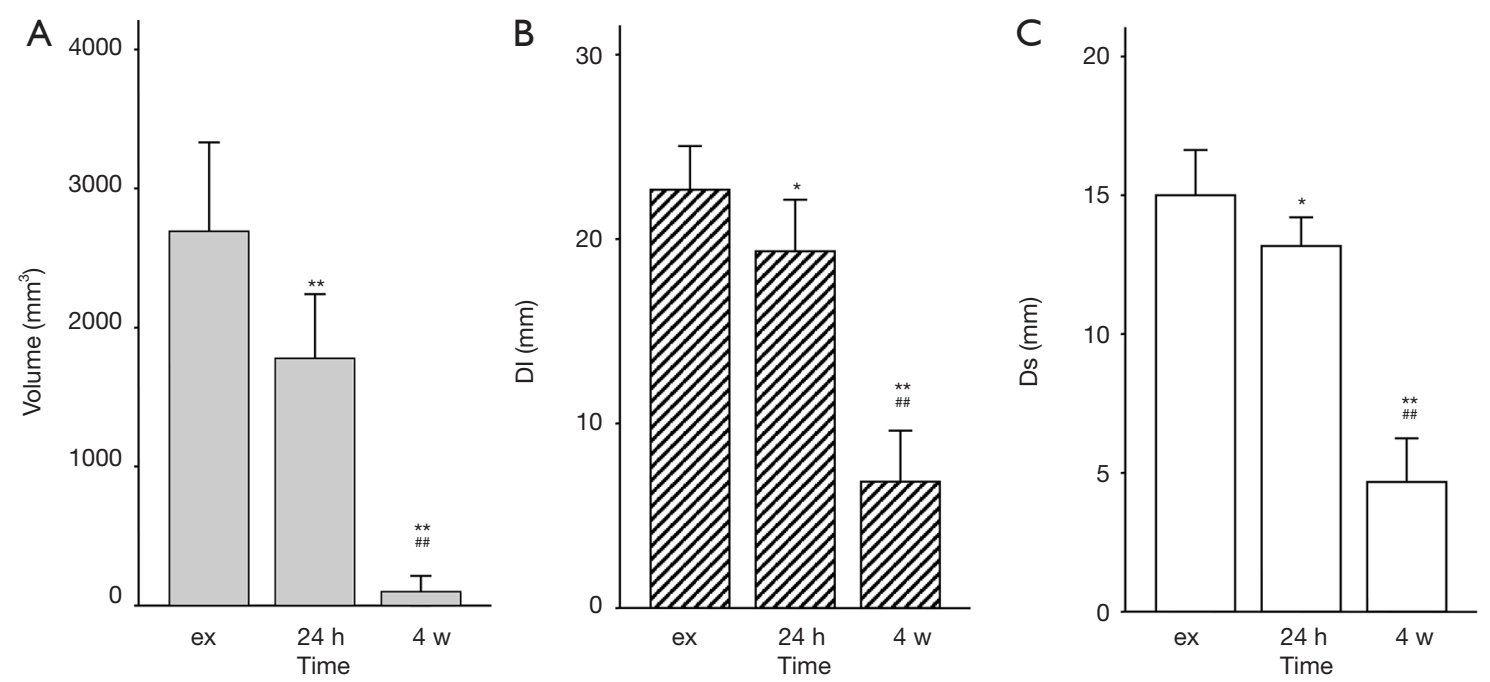

Figure 3 Macroscopic measurement changes of dissected lesions by microwave antenna via the working channel of the bronchoscope. Volume (A), Dl (B) and Ds (C) of lesions compared ex vivo, 24 hours post ablation in vivo, 4 weeks post ablation in vivo. Results are mean \pm SD (n=18, 6 in ex vivo and 12 in vivo). ${ }^{*} \mathrm{P}<0.05,{ }^{*} \mathrm{P}<0.01$ vs. ex group; ${ }^{* \#} \mathrm{P}<0.01$ vs. 24 h group. D1, long-axis diameter; Ds, short-axis diameter; ex, ex vivo; 24 h, 24 hours; 4 w, 4 weeks. 

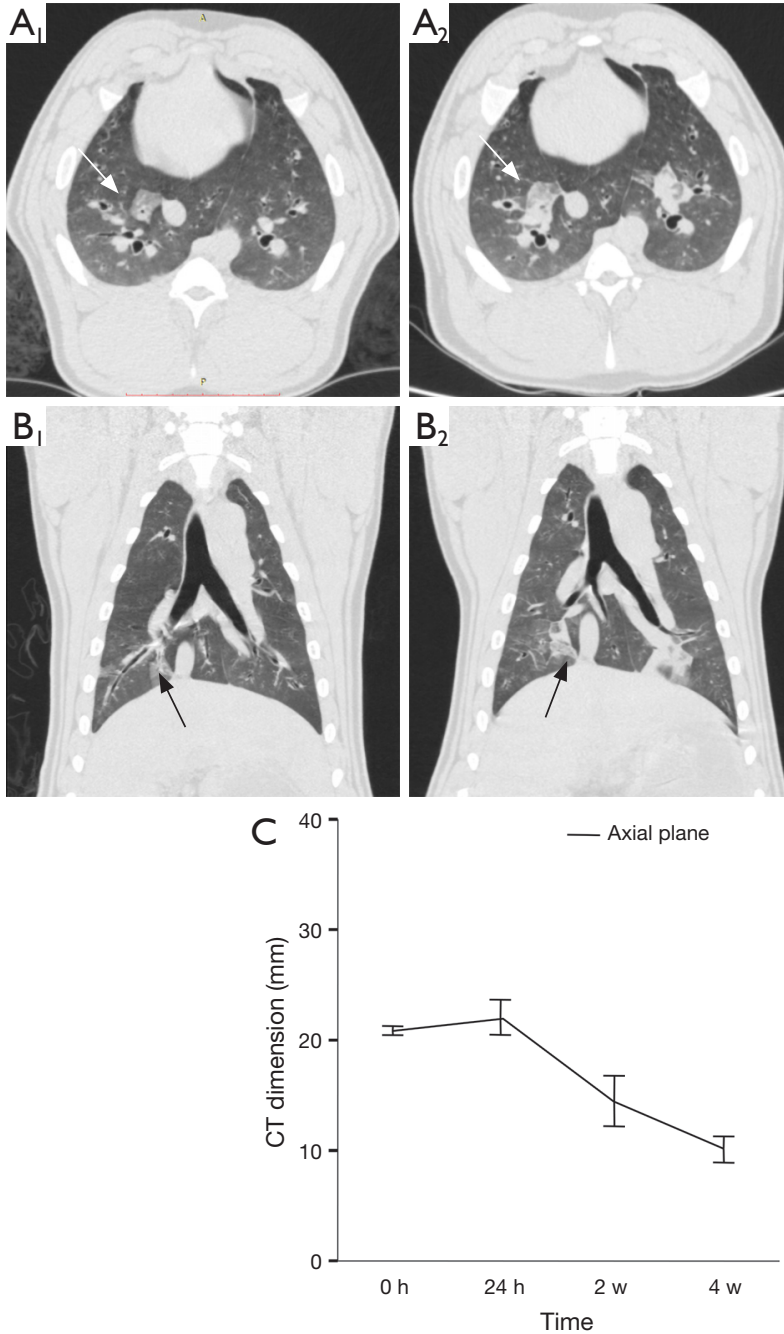
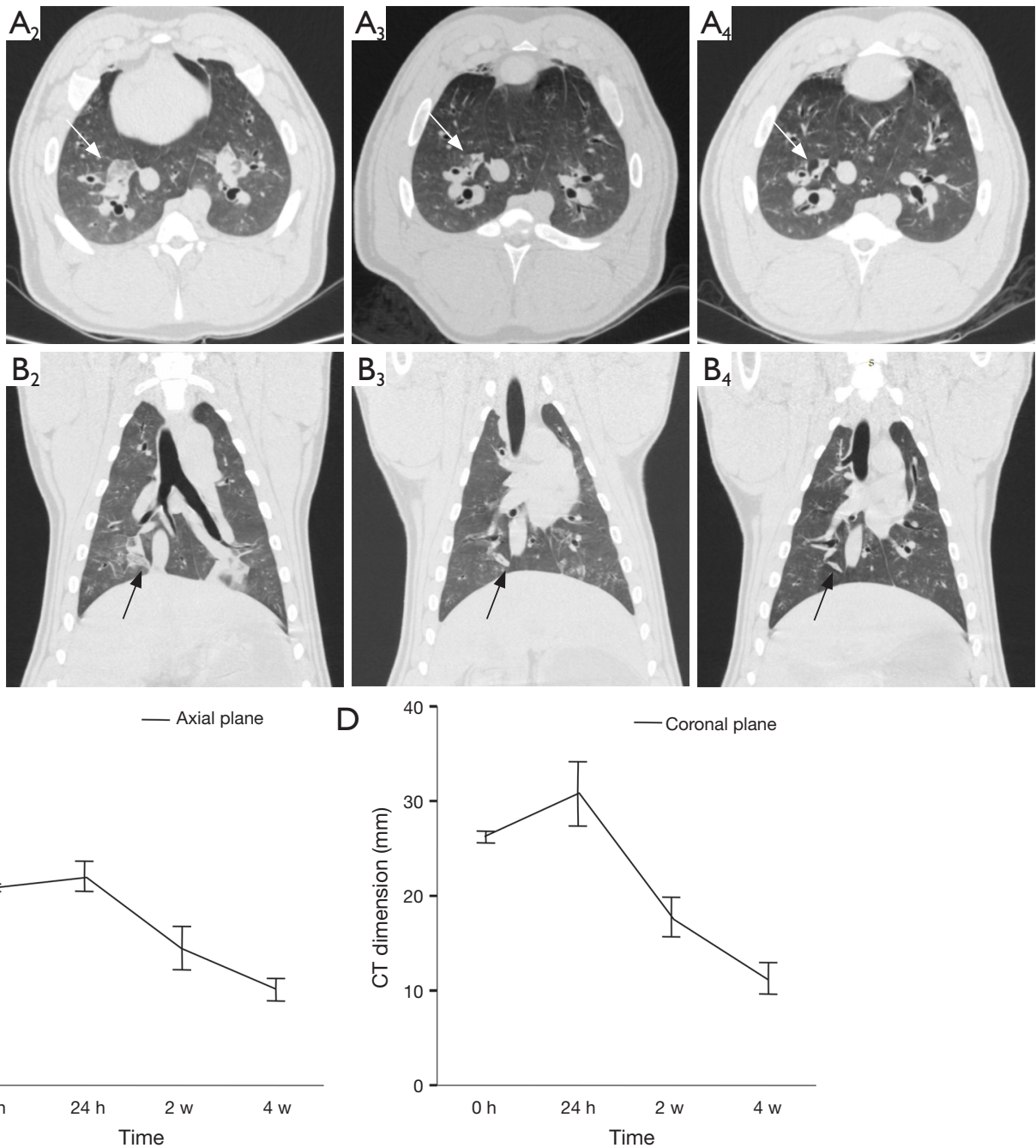

Figure 4 Representative CT findings of the ablated lesion (arrowhead) and the lesion dimension changes as measured using multiplanar reformatted CT images in the axial (A1-4) and coronal planes (B1-4) at different time points post ablation. (A1,B1) demonstrate GGO change immediately; (A2,B2) The changes became more solid with some hypoattenuation. (A3,B3) At two weeks, the changes appear solid. (A4,B4) The nodule at 4 weeks. (C,D) Measured changes in CT dimension over time in the axial and coronal planes respectively. CT, computed tomography; GGO, ground glass opacity.

cartilage still retained the original organization structure. The inner layer show signs of thermal damage but the tissue maintains a seemingly intact alveolar structure with little effusion. Using a TUNEL Assay, the epithelium of BM was almost completely apoptotic, but bronchial cartilage was still viable without any apoptosis. The inner layer of ablation center suggested that cells were almost completely apoptotic (Figure 5).

\section{Four weeks after ablation (group B)}

A circle of fibrous scar tissue was formed around the bronchus. The BM and cartilage were still intact and plenty of fibrous tissue could be seen in the ablation center. Using a Masson trichrome staining, the fibroblast cells or tissues underneath BM were seen, while the focal area of ablation is completely replaced by the fibroblast cells or tissues (Figure 6).

\section{Discussion}

During years prior, various percutaneous image-guided techniques for ablation have been developed for the treatment of lung tumors. The risk of complications was 

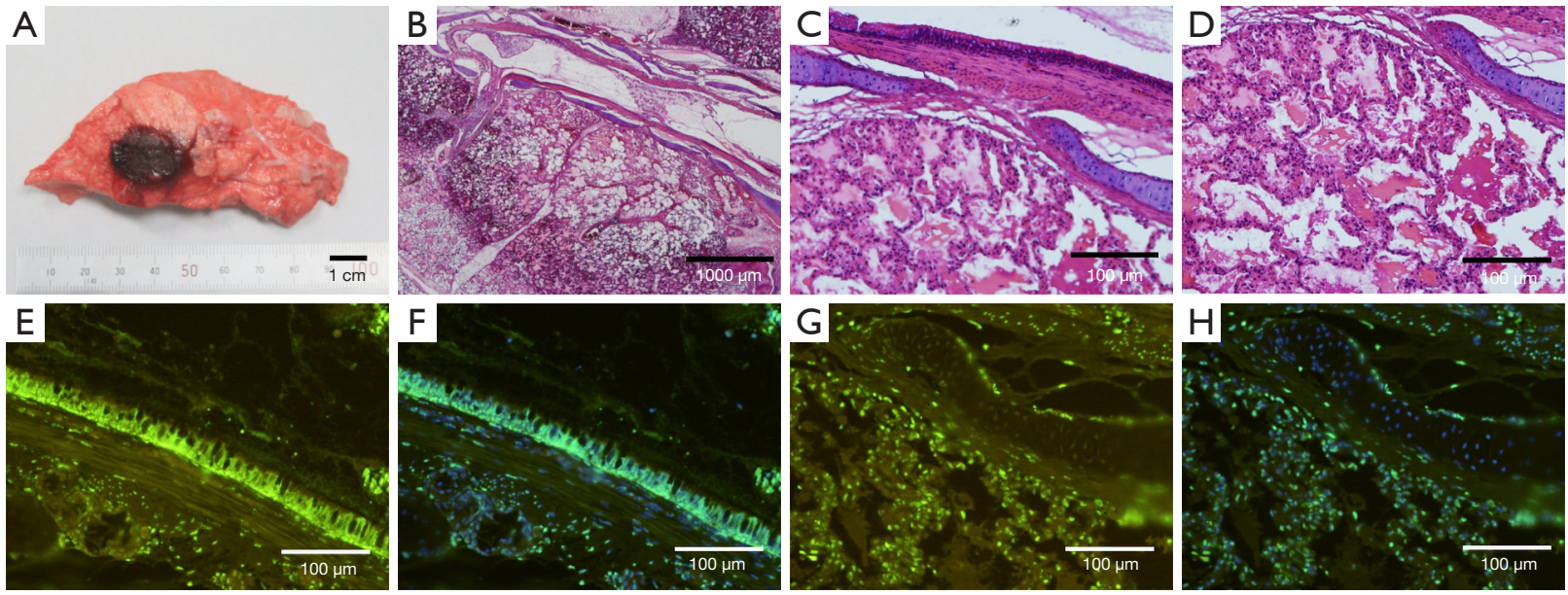

Figure 5 Representative histopathological findings: HE staining at 24 hours, apoptosis assayed with TUNEL staining at 24 hours after ablation. (A) The ablation zone at 24 hours was centered along the bronchus at the area of antenna insertion. (B) Around the bronchus, a succession of three concentric layers of tissue with different histological characteristics was shown. (C) The epithelium of the BM and cartilage still retain the original organization. (D) The cells in the innermost layer shown signs of thermal damage but the tissue maintained a seemingly intact alveolar structure. (E) The epithelium of BM is completely apoptotic (in green). (F) The epithelium of BM TUNEL (green) and DAPI (blue) merged. (G) Bronchial cartilage does not show apoptosis (in green) while the inner layer of the ablation center demonstrates apoptosis (in green). (H) TUNEL (green) and DAPI (blue) merged. Scale bars: $1 \mathrm{~cm}$ for A; 1,000 $\mu \mathrm{m}$ for B at 2× magnification; $100 \mu \mathrm{m}$ for C,D,E,F,G,H at 20× magnification. HE, hematoxylin-eosin; TUNEL, terminal deoxynucleotidyl transferase mediated dUTP nick end labeling; BM, bronchus mucosa; DAPI, 4',6-diamidino-2-phenylindole.
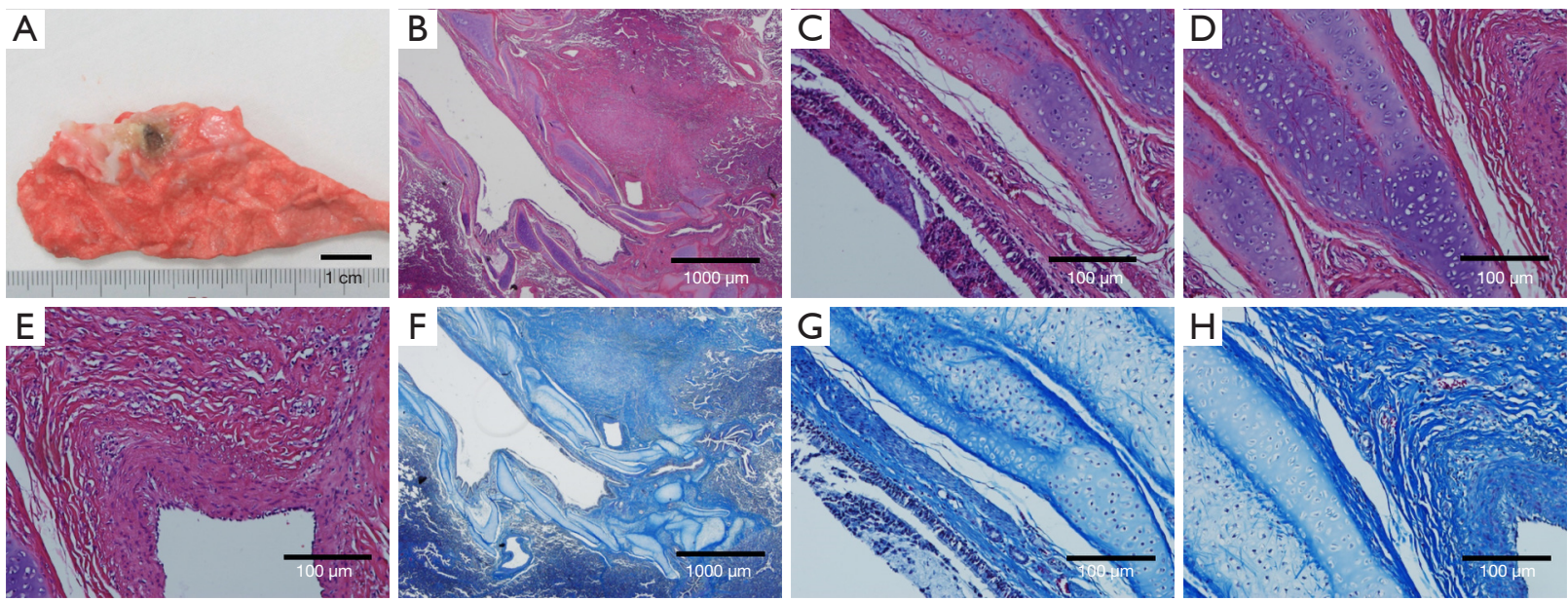

Figure 6 Representative histopathological findings: HE staining at 4 weeks, cell repair with Masson staining at 4 weeks post ablation. (A) Gross lung tissue sample showed a granular black lesion at 4 weeks. (B) Around the bronchus, a circle of fibrous scar tissue was formed. (C) The BM remained intact and some inflammatory exudate was visible in partial lumen. (D) Fibrous tissue seen in the center of the lesion. (E) Inflammatory cells infiltration. (F) Many of the fibroblast cells or tissues (blue areas) under repair. (G) The fibroblast cells or tissues underneath bronchial mucosa are shown. $(\mathrm{H})$ The focal area of ablation was completely replaced by the fibroblast cells or tissues. Scale bars: $1 \mathrm{~cm}$ for A; 1,000 $\mu \mathrm{m}$ for B, F at 2× magnification; $100 \mu \mathrm{m}$ for C,D,E, G,H at 20× magnification. HE, hematoxylin-eosin; BM, bronchus mucosa. 
related to the increased length of the lung traversed, similar to percutaneous lung biopsy $(15,23)$. The goal of using natural channels to reach the lesions and provide energy ablation safely has been demonstrated in the previous studies $(24,25)$. Tsushima et al. (26) showed that, bronchoscopy-guided RFA was safe and feasible in sheep lungs. Xie et al. (27) reported navigation bronchoscopyguided RFA for nonsurgical peripheral pulmonary tumors with success and a good safety profile.

There are three criteria to assign malignant nodules in lung detected on CT images: solid noncalcified nodules, pure ground-glass nodule and part-solid lung nodules (28). The component of ground-glass nodule on CT corresponds pathologically to alveolar septal thickness and contained air in the alveoli, namely lepidic growth preserving the existing alveolar structure of the cancer cells (29). As RFA is dependent on thermal conductivity, air-filled tumors tend to limit the size of the ablation zone. Microwave propagation is thought not to be similarly hindered by air and could be more easily applied to various types of tumors (30).

Flexible bronchoscopy-guided, water-cooled MWA is a relatively new technique that can avoid the risk of percutaneous operation and not be limited to air in the tumor. This is the first report showing the feasibility and safety of the flexible bronchoscopy-guided, water-cooled MWA in ex vivo and in vivo porcine lung.

The goals of our thermal ablation study were a $5-10 \mathrm{~mm}$ safety margin of apparently healthy tissue adjacent to tumor, avoiding injury to critical structures, and create a large ablation area quickly $(5,11,31)$. From prior work, it appears that thermal ablation works well, particularly in local control, for early-stage or small tumors $(<30 \mathrm{~mm})(32)$. In light of this, we originally set the range of ablation to the $20 \mathrm{~mm}$ radius with a $5 \mathrm{~mm}$ safety margin. In the temperature-rising curve of ex vivo lung, $80 \mathrm{~W}$ for $5 \mathrm{~min}$ extended the coagulation necrosis temperature to the 20 $\mathrm{mm}$ radius. During the in vivo live model, using the same $80 \mathrm{~W}$ for 5 minutes, the range was decreased sharply far from $20 \mathrm{~mm}$ radius. Respiratory motion and vascular factor ("heat-sink" caused by pulmonary blood flow) were likely a leading cause.

No serious complications occurred. CT imaging characteristics of the ablation zone were consistent with a GGO immediately after ablation illustrating the effectiveness of ablation, similar to percutaneous ablation (33). The lesion gradually changed into nodules with persistent reduction in size.

The limitations of previous clinical RFA studies done near the trachea are theoretically influenced by the trachea wall thickness (34). Our pathology at 24 hours also really found that there was no apoptosis in bronchus cartilage, which possibly affect the energy deposition of ablation. But electromagnetic energy field of MWA could still cause coagulation necrosis, similar to percutaneous ablation (35), in lung parenchyma around bronchus cartilage. Compared with RFA, the advantage of MWA is a demonstrated greater ablation range. Pathology at 4 weeks showed that the intact preservation of bronchus cartilage was helpful to repair the ablation area and reduce the production of unnecessary inflammation, especially obstructive inflammation.

This study has several limitations. First, our animal model was using normal healthy tissue lacking a tumor model. The properties of lung tumors are different from the properties of normal lung parenchyma. However, our experiment made it clear that MWA could produce ablation effect in air-filled normal lung tissue, which could mimic air-filled ground glass pulmonary neoplasms (36), the main objective of MWA. Second, although no major complications were observed during this study, the number of animal subjects and ablations done limit any ability to absolutely comment on safety. Third, the range of ablations under bronchoscopy were far from ideal percutaneous and more attempts need to be made and improved. Artificial atelectasis (e.g., target bronchial balloon catheter block) and ventilation reduction strategy can be tried. Fourth, the realtime temperature measurement and ablation range were not real-time monitor during the endobronchial operation. We propose a preliminary solution that setting real-time temperature measuring points on the catheter different distance from the tip of antenna. In summary, we have successfully used a flexible bronchoscopy-guided microwave antenna to perform ablation in the lung via the natural bronchial lumen in an animal and it appears to be effective and safe.

\section{Acknowledgments}

Funding: This work was supported by The National Key R\&D Program of China (2017YFC0112700); Clinical Research Plan of SHDC (16CR3007A); Scientific Research Program by Science and Technology Commission of Shanghai Municipality (16441900702, 15411964300).

\section{Footnote}

Conflicts of Interest: The authors have no conflicts of interest 
to declare.

Ethical Statement: The authors are accountable for all aspects of the work in ensuring that questions related to the accuracy or integrity of any part of the work are appropriately investigated and resolved. This study was approved by the Ethics Committee at Shanghai Chest Hospital (Shanghai, China) (KS1610).

\section{References}

1. Torre LA, Bray F, Siegel RL, et al. Global cancer statistics, 2012. CA Cancer J Clin 2015;65:87-108.

2. Kim ES, Kim YT, Kang CH et al. Prevalence of and risk factors for postoperative pulmonary complications after lung cancer surgery in patients with early-stage copd. Int J Chron Obstruct Pulmon Dis 2016;11:1317-26.

3. Diwanji TP, Mohindra P, Vyfhuis M, et al. Advances in radiotherapy techniques and delivery for non-small cell lung cancer: benefits of intensity-modulated radiation therapy, proton therapy, and stereotactic body radiation therapy. Transl Lung Cancer Res 2017;6:131-47.

4. Zheng X, Schipper M, Kidwell K et al. Survival outcome after stereotactic body radiation therapy and surgery for stage i non-small cell lung cancer: A meta-analysis. Int J Radiat Oncol Biol Phys 2014;90:603-11.

5. Ahmed M, Brace CL, Lee FT, et al. Principles of and advances in percutaneous ablation. Radiology 2011;258:351-69.

6. Rim CH, Seong J. Application of radiotherapy for hepatocellular carcinoma in current clinical practice guidelines. Radiat Oncol J 2016;34:160-7.

7. Yuan Z, Wang Y, He ML, et al. Successful ablation for pulmonary artery tumor thrombosis more than $5 \mathrm{~cm}$ with massive hepatocellular carcinoma and multiple pulmonary metastases. Transl Cancer Res 2018;7:456-61.

8. Brace CL. Radiofrequency and microwave ablation of the liver, lung, kidney, and bone: What are the differences? Curr Probl Diagn Radiol 2009;38:135-43.

9. Wright AS, Sampson LA, Warner TF, et al. Radiofrequency versus microwave ablation in a hepatic porcine model. Radiology 2005;236:132-9.

10. Yu J, Liang P, Yu X et al. A comparison of microwave ablation and bipolar radiofrequency ablation both with an internally cooled probe: Results in ex vivo and in vivo porcine livers. Eur J Radiol 2011;79:124-30.

11. Goldberg SN, Gazelle G, Mueller P. Thermal ablation therapy for focal malignancy: A unified approach to underlying principles, techniques, and diagnostic imaging guidance. AJR Am J Roentgenol 2000;174:323-31.

12. Ierardi AM, Giorlando F, Piacentino F, et al. Factors predicting outcomes of microwave ablation of small hepatocellular carcinoma. Radiol Med 2017;122:81-7.

13. Acksteiner C, Steinke K. Percutaneous microwave ablation for early-stage non-small cell lung cancer (nsclc) in the elderly: A promising outlook. J Med Imaging Radiat Oncol 2015;59:82-90.

14. Carberry GA, Nocerino E, Mason PJ, et al. Pulmonary microwave ablation near the heart: Antenna positioning can mitigate cardiac complications in a porcine model. Radiology 2017;282:892-902.

15. Vogl TJ, Naguib NN, Lehnert T, et al. Radiofrequency, microwave and laser ablation of pulmonary neoplasms: Clinical studies and technical considerations--review article. Eur J Radiol 2011;77:346-57.

16. Wiener RS, Schwartz LM, Woloshin S, et al. Populationbased risk for complications after transthoracic needle lung biopsy of a pulmonary nodule: An analysis of discharge records. Ann Intern Med 2011;155:137-44.

17. Wang Memoli JS, Nietert PJ, Silvestri GA. Metaanalysis of guided bronchoscopy for the evaluation of the pulmonary nodule. Chest 2012;142:385-93.

18. Ferguson J, Egressy K, Schefelker R, et al. Bronchoscopically-Guided Microwave Ablation in the Lung. Chest 2013;144:87A.

19. Simon CJ, Dupuy DE, Mayo-Smith WW. Microwave ablation: Principles and applications. Radiographics 2005;25:S69-83.

20. Ridge CA, Huang J, Cardoza S, et al. Comparison of multiplanar reformatted ct lung tumor measurements to axial tumor measurement alone: Impact on maximal tumor dimension and $\mathrm{t}$ stage. AJR Am J Roentgenol 2013;201:959-63.

21. Rychel AL, Swalla BJ. Anterior regeneration in the hemichordate ptychodera flava. Dev Dyn 2008;237:3222-32.

22. Yamamoto M, Murata K, Kiriu T, et al. Acute fibrinous and organizing pneumonia with myelodysplastic syndrome: Corticosteroid monotherapy led to successful ventilator weaning. Intern Med 2016;55:3155-9.

23. Hiraki T, Tajiri N, Mimura H, et al. Pneumothorax, pleural effusion, and chest tube placement after radiofrequency ablation of lung tumors: Incidence and risk factors. Radiology 2006;241:275-83.

24. Krimsky WS, Pritchett MA, Lau KKW. Towards an optimization of bronchoscopic approaches to the diagnosis 
and treatment of the pulmonary nodules: A review. J

Thorac Dis 2018;10:S1637-44.

25. Vieira T, Stern JB, Girard P, et al. Endobronchial treatment of peripheral tumors: Ongoing development and perspectives. J Thorac Dis 2018;10:S1163-7.

26. Tsushima K, Koizumi T, Tanabe T, et al. Bronchoscopyguided radiofrequency ablation as a potential novel therapeutic tool. Eur Respir J 2007;29:1193-200.

27. Xie F, Zheng X, Xiao B, et al. Navigation bronchoscopyguided radiofrequency ablation for nonsurgical peripheral pulmonary tumors. Respiration 2017;94:293-8.

28. MacMahon H, Naidich DP, Goo JM, et al. Guidelines for management of incidental pulmonary nodules detected on ct images: From the fleischner society 2017. Radiology 2017;284:228-43.

29. Aokage K, Miyoshi T, Ishii G, et al. Influence of ground glass opacity and the corresponding pathological findings on survival in patients with clinical stage i non-small cell lung cancer. J Thorac Oncol 2018;13:533-42.

30. Pua BB, Thornton RH, Solomon SB. Ablation of pulmonary malignancy: Current status. J Vasc Interv

Cite this article as: Yuan HB, Wang XY, Sun JY, Xie FF, Zheng XX, Tao GY, Pan L, Hogarth DK. Flexible bronchoscopy-guided microwave ablation in peripheral porcine lung: a new minimally-invasive ablation. Transl Lung Cancer Res 2019;8(6):787-796. doi: 10.21037/tlcr.2019.10.12
Radiol 2010;21:049.31.

31. Sidoff L, Dupuy DE. Clinical experiences with microwave thermal ablation of lung malignancies. Int J Hyperthermia 2017;33:25-33.

32. Klapper JA, Hittinger SA, Denlinger CE. Alternatives to lobectomy for high-risk patients with early-stage nonsmall cell lung cancer. Ann Thorac Surg 2017;103:1330-9.

33. Wolf FJ, Grand DJ, Machan JT, et al. Microwave ablation of lung malignancies: Effectiveness, ct findings, and safety in 50 patients. Radiology 2008;247:871-9.

34. Koizumi T, Tsushima K, Tanabe T, et al. Bronchoscopyguided cooled radiofrequency ablation as a novel intervention therapy for peripheral lung cancer. Respiration 2015;90:47-55.

35. Yamamoto A, Nakamura K, Matsuoka T, et al. Radiofrequency ablation in a porcine lung model: Correlation between ct and histopathologic findings. AJR Am J Roentgenol 2005;185:1299-306.

36. Koo CW, Miller WT, Kucharczuk JC. Focal groundglass opacities in non-small cell lung carcinoma resection patients. Eur J Radiol 2012;81:139-45. 